\title{
The 3 Rs of Stroke Biology: Radial, Relayed, and Regenerative
}

\author{
S. Thomas Carmichael ${ }^{1}$
}

Published online: 24 November 2015

(C) The American Society for Experimental NeuroTherapeutics, Inc. 2015

\begin{abstract}
Stroke not only causes initial cell death, but also a limited process of repair and recovery. As an overall biological process, stroke has been most often considered from the perspective of early phases of ischemia, how these inter-relate and lead to expansion of the infarct. However, just as the biology of later stages of stroke becomes better understood, the clinical realities of stroke indicate that it is now more a chronic disease than an acute killer. As an overall biological process, it is now more important to understand how early cell death leads to the later, limited recovery so as develop an integrative view of acute to chronic stroke. This progression from death to repair involves sequential stages of primary cell death, secondary injury events, reactive tissue progenitor responses, and formation of new neuronal circuits. This progression is radial: from the tissue that suffers the infarct secondary injury signals, including free radicals and inflammatory cytokines, radiate out from the stroke core to trigger later regenerative events. Injury and repair processes occur not just in the local stroke site, but are also triggered in the connected networks of neurons that had existed in the stroke center: damage signals are relayed throughout a brain network. From these relayed, distributed damage signals, reactive astrocytosis, inflammatory processes, and the formation of new connections occur in distant brain areas. In short, emerging data in stroke cell death studies and the development of the field of stroke neural repair now indicate a continuum in time and in space of progressive events that can be considered as the 3 Rs of stroke biology: radial, relayed, and regenerative.
\end{abstract}

\section{S. Thomas Carmichael \\ SCarmichael@mednet.ucla.edu}

1 Departments of Neurology and Neurobiology, David Geffen School of Medicine at UCLA, Los Angeles, CA, USA
Key Words Axonal sprouting · Neurogenesis · Angiogenesis $\cdot$ Excitotoxicity $\cdot$ Astrocyte $\cdot$ Oligodendrocyte progenitor

\section{Introduction}

Stroke is declining as a cause of death in the USA. Two years ago stroke slipped from the third leading cause of death to the fourth [1], and in 2015 it fell to the fifth leading cause of death [2]. Even with improving treatments for stroke risk factors, and a possible decline in stroke incidence from risk factor control, mortality from stroke will decline faster than the incidence [3]. This fact means that stroke is morphing into a chronically disabling disease. Neuroscience must more fully integrate the currently dual aspects of basic stroke research, those of cell death and of neural repair, into a more integrated whole so as to translate candidate biological therapies into novel treatments. This review provides a description of the events in stroke as they develop from the infarct to adjacent and distant tissue, and as they move from death to injury to repair: radial, relayed, and regenerative (3Rs) aspects of stroke. The final regenerative section discusses axonal sprouting and the formation of new connections, in joining with other reviews in this Neural Circuits themed issue.

\section{Radial Stroke: Zones of Damage}

Stroke can be conceived as a site of complete cellular death surrounded by tissue reorganization. This concept was first suggested for the progression of early damage in acute stroke [4] but has even greater meaning in the changes of tissue repair and recovery, such as distinct zones of reactive astrocytes and dendritic spine changes. 


\section{The Infarct Core}

Ischemic stroke starts as an acute loss of blood flow to a region of the brain. This precipitates a cascade of direct damage in neurons, astrocytes, blood vessels (endothelial cells and pericytes), oligodendrocytes, microglia, and oligodendrocyte precursor cells. These 7 cell types can be principally considered the parenchyma of the brain. Ischemic cell death has, of course, been best studied in neurons $[5,6]$. The initial loss of blood supply causes the most energetically expensive neuronal function to collapse: the maintenance of resting membrane potential. Neurons depolarize as a result, seen as the anoxic depolarization in extracellular recordings. Neurons release glutamate as they depolarize, which causes further depolarization of neighboring neurons, further glutamate release, and a positive feedback cycle. Glutamate, acting through the $\mathrm{N}$ methyl-D-aspartate receptor, induces massive intracellular calcium entry. Calcium also enters through voltagedependent calcium channels as the neuron depolarizes. A major calcium sink in the neuron is the mitochondrion, which swells as it buffers calcium and becomes dysfunctional. Calcium-mediated proteolysis, lipolysis, and DNA degradation lead to necrotic cell death. There are other molecular actors in this initial neuronal cell death, including free radical formation in the disordered mitochondria. In total, this is the process of neuronal excitotoxicity, first prominently described in stroke in the late 1980s and the motive force behind many of the stroke clinical trials of the 1990s [7]. The initial excitotoxic death of neurons is quickly irreversible and a reanalysis of preclinical animal modeling data and the experience of the failed stroke trials indicate that the initial phase of neuronal cell death is quite likely beyond the reach of clinical medicine as a therapeutic target.

The brain contains at least 6 other cell types than neurons. How these die in stroke, and how this damage leads to dysfunction and partial recovery is less clear. In vitro data indicate that neurons are the most sensitive to oxygen/glucose deprivation, followed by endothelial cells, astrocytes, and microglia [8]. As with neurons, glutamate signaling may play a role in oligodendrocyte cell death after stroke, through both $N$-methyl-D-aspartate and $\alpha$-amino-3-hydroxy-5-methyl-4-isoxazolepropionic acid subtypes of the glutamate receptor [9]. In rodent stroke models, oligodendrocytes survive longer than neurons [10]. Astrocytes die at a somewhat later period in the same ischemic stimulus than neurons $[8,11]$, and do so through mechanisms that involve swelling, adenosine triphosphate release and signaling through adenosine triphosphate receptors $\left(\mathrm{P}^{2} \mathrm{Y}_{1}\right.$ purinergic receptors [12]). With this differential sensitivity in mind, even in the short term in vivo, hours after the infarct, all cell types in the stroke core die. They do so with slightly different time scales and cellular mechanisms but die nonetheless. Tissue repair and recovery depend, in large part, on what happens adjacent to the stroke core - the radial effects of the ischemic stimulus on the surrounding brain region.

\section{Peri-infarct Tissue}

Peri-infarct is a regional term - the topographic description of tissue that is next to the stroke core and has experienced partial damage. This is not the same term as the stroke "penumbra". The penumbra was originally defined physiologically as the region of electrical silence in initially intact but hypoxic tissue at the onset of ischemia [13]. The ischemic penumbra has come to mean the brain tissue that is not initially damaged by stroke but will progress to infarction over time if there is no therapy initiated. In the emergency department and clinical stroke unit, medical care is mobilized to image the stroke penumbra and maximize resources to save it, though intra-arterial delivery of a clot buster or stent, as examples [14]. Human imaging and rodent preclinical stroke models indicate that the ischemic penumbra will die and become part of the stroke core in roughly 2 days [15]. In other words, in the acute period after stroke the core dies and the penumbra has 2 fates: it will either die and become part of the stroke core, or be salvaged by therapy and survive. In the former case, the periinfarct tissue borders the now enlarged stroke core. In the latter the salvaged penumbra is now part of the peri-infarct tissue. From the perspectives of the biology of tissue repair and the time course of recovery, the debate over the penumbra is moot: this is really a progression of the process of initial ischemic cell death. However, the mechanisms of cell death in the penumbra have important roles as triggers for neural repair and recovery, and do so likely via radial transmission to peri-infarct tissue.

Delayed cell injury and death in the penumbra occur with inflammation and free radical generation. The partial blood supply in the penumbra activates endothelial cells to localize a tremendous neutrophil and macrophage influx $[10,16]$. Microglia are activated within minutes in injured stroke tissue [17], and, unlike in the stroke core, survive [10]. Astrocytes activate into a reactive phenotype in days after stroke and produce cytokines, matrix metalloproteinases, and chondroitin sulfate proteoglycans $[18,19]$. These 3 cell types release cytokines and produce free radicals that produce secondary damage, modify the extracellular matrix, and also generate the signals for neural repair.

\section{Radial Stroke: Triggers for Neural Repair}

The progression of brain ischemia over time from the infarct core to the penumbra and peri-infarct tissue involves secondary injury cascades. These cascades involve reperfusion injury in damaged cells, which causes free radical production, and the activation of astrocytes and white blood cells, which release cytokines and free radicals, and the production of synchronized neuronal activity. This is a radial effect of stroke that triggers neural repair. Free radicals generated in penumbra and in peri-infarct tissue are part of the stimulus for neurogenesis [20,21]; cytokines activate astrocytes, promote angiogenesis, and induce axonal sprouting [19, 22, 23]; and 
synchronized neuronal activity induces axonal sprouting and the formation of new connections (Fig. 1) [24].

Inflammatory cytokines are released by activated microglia (early), invading neutrophils (early), and macrophages (later) $[25,26]$. These can directly stimulate aspects of neural repair [27], and also induce an activated state in brain endothelial cells and astrocytes. Activated astrocytes and endothelial cells then further produce molecules that induce or alter the brain's reparative response $[19,28]$. Several cytokines from these cells influence a stem cell response to stroke. Activated blood vessels in peri-infarct tissue secrete stromal-derived factor 1 (SDF-1), a cytokine that is tropic to immature neurons as they migrate to areas of damage after stroke [29, 30]. Chemokine (C-C motif) ligand 2, leukemia inhibitory factor, and tumor necrosis factor (TNF)- $\alpha$ are also inflammatory signaling molecules that are released by astrocytes and microglia/ macrophages after stroke, induce distinct stages of normal neurogenesis, and have been implicated in poststroke neurogenesis [27, 31, 32]. Activated astrocytes and microglia release other cytokines and chemokines that participate in poststroke neurogenesis [27, 30, 33].

Inflammatory cytokines also participate in poststroke axonal sprouting: the formation of new connections. As discussed below, axonal sprouting after stroke induces the formation of new connections in peri-infarct cortex among motor, premotor, and somatosensory cortical areas. These connections causally mediate motor recovery. The transforming growth factor (TGF) superfamily of cytokines contains several molecules that stimulate poststroke axonal sprouting. Bone morphogenic protein 7 stimulates dendritic growth in neurons and promotes behavioral recovery $[34,35]$. The TGF- $\beta$ family member growth differentiation factor 10 (GDF10) is induced in peri-infarct cortex after stroke and is a potent stimulant for axonal sprouting and functional recovery [36]. Activation of astrocytes in peri-infarct cortex, in part through inflammatory cytokines, induces molecules that block axonal sprouting, such as chondroitin sulfate proteoglycans or ephrin-A5 [37-39]. Thus, peri-infarct inflammatory signals control both the induction of axonal sprouting and the induction of axonal growth inhibitors that block axonal sprouting. As discussed below, the local interplay of progrowth and growth-inhibiting molecular systems determines the extent and pattern of axonal sprouting and recovery (Fig. 1).

In addition to cytokines and other inflammatory molecules, stroke generates free radicals in the infarct core and peri-infarct tissue. This process occurs through the initial ischemia itself, through reperfusion and through inflammatory cell release [40]. Free radicals have potent biological effects because they are freely diffusible [41], can have a long tissue half-life [41, 42], and activate many downstream molecular processes. This last element of free radical action is a crucial distinction in the radial nature of stroke. In the infarct core, free radical generation produces tissue damage and cell death. In the peri-infarct cortex, free radical generation activates downstream signaling cascades in cells that will survive. These intracellular cascades mimic the signaling of growth factors [20, 43]. Free radicals also directly activate neural stem cells through induction of growth factor signaling pathways [20]. Through these systems, free radical generation after stroke is one of the signals of poststroke neurogenesis [21, 44]. Immature neurons in the largest germinal matrix, the subventricular zone (SVZ), respond to the stroke, migrate to the area of damage, and may participate in

\section{Continuum of Stroke}

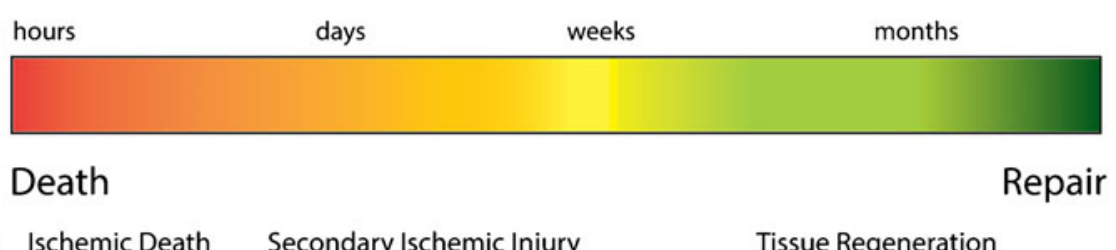

Stages Ischemic Death Secondary Ischemic Injury

Tissue Regeneration

Processes Excitoxicity, Apoptosis Inflammatory Cell Infiltration Structural Brain Reorganization

Regenerative Events
Angiogenesis

Neurogenesis

Gial Progenitor Responses

Axonal Sprouting
Circuits

Molecules
Neuronal Circuit Excitability Changes New Connections Loss of Cortical Sensorimotor Maps Remapping of Cortical Sensorimotor Function

Cytokine/Chemokine Production Free Radical Generation
Molecular Axonal Growth Program

Fig. 1 Stroke progresses in time from initial to secondary injury and to tissue repair as a continuum in which the initial pathological events trigger later regenerative events 
neural repair [30, 45]. Blocking free radical generation, such as with an antioxidant, reduces poststroke neurogenesis [21]. It appears that free radical generation in peri-infarct tissue is one of the signals to multipotent neural stem cells and their progeny in the SVZ, together with other cytokines and growth factors activated in peri-infarct cortex [27, 30, 45].

In the early stages after stroke, peri-infarct tissue exhibits synchronized electrical activity. Within the first several days after stroke, peri-infarct spreading depressions sweep through cortex. These are synchronized and long-duration neuronal depolarizations that produce a substantial metabolic demand on peri-infarct tissue. This demand may be so great that the tissue may die; in other words, peri-infarct spreading depressions may push a region of penumbra into a region of stroke cell death or core $[40,46]$. However, peri-infarct spreading depressions also occur in tissue that will survive [47]. Though peri-infarct spreading depressions represent a powerful envelope of synchronized neuronal discharges. These sweeps of neuronal activity do not correlate with later regenerative events, such as axonal sprouting [24], and the spreading depression may be conceptualized as a radial injury event from the infarct to the adjacent tissue. Peri-infarct spreading depressions are followed in the first several days after stroke by synchronized low-frequency neuronal discharges across periinfarct cortex and connected (contralateral) cortical areas, which occur at $<0.1 \mathrm{~Hz}[24,48]$. This low-frequency synchronized neuronal activity is a transition from acute stroke injury and death to longer-term repair, as discussed below. Thus synchronized neuronal activity after stroke is not just a bridge between damage to repair, but also one of the early events to be relayed from the damaged tissue to more distant connected sites, such as contralateral cortex [24]. Synchronized neuronal activity after stroke might be classified into "radial" or "relayed" but has been placed in this section to align with the other major synchronized neuronal activity event after stroke, peri-infarct spreading depressions.

Peri-infarct synchronized neuronal activity is a trigger for axonal sprouting and the formation of new connections after stroke [24]. It occurs in lesion conditions, such as stroke, that trigger axonal sprouting, and not lesion conditions, such as equivalently sized aspiration of cortex, that do not induce axonal sprouting. Blocking peri-infarct synchronized neuronal activity blocks poststroke axonal sprouting in peri-infarct cortex and in contralateral cortex to the infarct [23]. In serving as a trigger, this synchronized neuronal activity in peri-infarct cortex after stroke resembles similar activity patterns in the formation of brain connections during development in the retina, hippocampus, and cortex [49-51]. Synchronized neuronal activity may activate a downstream molecular program for neuronal growth, or may stimulate the co-activation of many synapses in a particular region of neurons, stimulating a Hebbian plasticity and synaptic sprouting after stroke, as it does in the developing brain.

\section{Radial Stroke: Tissue Reorganization}

In the days to weeks after stroke, the peri-infarct cortex loses its initial response to afferent inputs and then gradually regains this response, with new locations of sensory or motor body maps [52, 53]. This process of an initial loss of sensory, motor, or language representation in peri-infarct and connected areas is seen in humans and in animal models of stroke [54-56]. The best recovery in human stroke occurs when motor, sensory, or language function is re-mapped into peri-infarct and connected regions $[54,55]$. This process of remapping occurs with widespread changes in the location and temporal flow of neuronal excitability, visualized with voltage-sensitive dye responses with optical imaging $[52,53]$. There is an initial downscaling of peri-infarct responsiveness in neurons in the first week after stroke, and then a differential return of neuronal activation within peri-infarct cortex [57]. These data identify alterations at the macrolevel in neuronal networks after stroke - changes in cortical map representation and in the temporal pattern of actions of large ensembles of neurons in periinfarct cortex.

The local physiology of network connections in peri-infarct cortex is also altered after stroke. Stroke causes an increase in $\gamma$-aminobutyric acid (GABA)ergic tonic inhibition in periinfarct neurons [58]. Tonic GABA inhibition is distinct from phasic, or synaptic, GABA inhibition. Phasic or synaptic GABA action is the common mechanism of intracortical inhibition that is seen in measures, like paired pulse inhibition in both transcranial magnetic stimulation and in cell electrophysiology. Phasic GABA inhibition is fast and is communicated by direct synaptic contact in a precise, circuit-specific way. By contrast, tonic GABA inhibition is a slow inhibitory current that is mediated, in part, by levels of extracellular GABA in the milieu surrounding a group of neurons. This current establishes the threshold for action potential firing of a neuron [59]. After stroke, reactive astrocytes have a downregulation of GABA uptake mechanisms, leading to increased extracellular GABA levels. This increases the baseline inhibitory tone in pyramidal or excitatory neurons. This process of increased tonic inhibition is initially neuroprotective. Blocking tonic GABA inhibition in the first several days after stroke causes an increase in ischemic cell death. However, the increased in tonic GABA inhibition persists long after the threat of ischemic cell death and by persisting into the period of recovery, the increased inhibition of neurons in peri-infarct tissue contributes to impaired circuit function in this area. Blocking tonic GABA inhibition after the first several days from the stroke promotes motor recovery [58]. This is a tractable pharmacological therapy for stroke recovery.

Stroke induces a change in neuronal morphology in the regions of these altered neuron electrophysiological responses. Dendritic spines form the synaptic contacts of cortical pyramidal neurons. These are mostly stable in the adult 
cortex $[60,61]$, although they remodel in response to loss of afferent inputs or learning [62-64]. After stroke, there is initially a net loss of dendritic spines in peri-infarct cortex in the first week after stroke $[52,65]$. This occurs in regions with normal blood flow [65], indicating that this loss is due to neuronal network damage from loss of axonal connections and not due to partial ischemia in peri-infarct regions. After this loss of connections, peri-infarct cortex within $1 \mathrm{~mm}$ of the infarct in the mouse recovers synaptic connections back to baseline [65], whereas neurons in regions 1-2 $\mathrm{mm}$ away from the infarct gain synaptic connections compared with control [65], indicating these neurons form new connections after stroke. This can be seen also as supernumerary axons exiting the neuronal cell body [66]. The actual branches of dendrites also remodel after stroke, with retraction and growth that is maximal 2 weeks after the infarct and occurs most prominently within $200 \mu \mathrm{m}$ of the infarct core [67]. Stroke also induces new axonal connections from neurons in peri-infarct cortex, which are initiated in the first week after stroke and are reliably present 1 month after stroke [39, 68, 69], particularly in the superficial cortical layers. These data indicate that stroke causes alterations in neuron morphology and the network of connections in peri-infarct tissue as result of the loss of connections with the infarct core; it occurs over specific time scales and in identified spatial zones within peri-infarct tissue radial to the stroke (Fig. 2).

Within peri-infarct tissue additional anatomical zones are formed by reactive astrocytes. Reactive astrocytes are not one homogenous type of cell but instead display distinct morphological and functional properties in zones that extend away from the infarct or central nervous system injury. This has been best detailed in the injured spinal cord and in normal brain, where distinct morphologies of reactive astrocytes indicate likely different zones of tissue repair and recovery [70], or of distinct circuit function [71]. In stroke, distinct types of reactive astrocytes are also present. The border of the stroke is formed by reactive astrocytes that have evolved into a bipolar morphology from their normal multipolar state and secrete large amounts of chondroitin sulfate proteoglycans [18]. This zone close to the infarct appears to be the only region where there is true astrocyte proliferation after stroke [72, 73]. Ablation of these border astrocytes after stroke and other injuries results in increased inflammation and worsening lesion size [74]. This protective function of reactive astrocytes fits well with the transcriptional profile of reactive astrocytes after stroke compared with reactive astrocytes after an inflammatory stimulus. Reactive astrocytes after a middle cerebral artery occlusion model of stroke showed a gene expression profile that was neuroprotective, with neurotrophic factors and cytokines, such as brain-derived neurotrophic factor and connective tissue growth factor, whereas after inflammatory stimulation reactive astrocytes activated complement, interferon, and other inflammatory and synapse-destruction pathways [75]. At distances more removed from the stroke in peri-infarct cortex, astrocytes still "react" to the stroke, and display increased glial fibrillary acidic protein expression but retain their normal multipolar morphology $[18,76]$. Astrocytes show distinct molecular properties by brain region, in such systems as sonic hedgehog signaling, glutamate uptake, and extracellular recognition and patterning molecules [71, 77]. It is likely that these distinct astrocyte subtypes will persist in the reactive astrocytosis after stroke and lead to zones in which scarforming molecules and other axonal growth inhibitors are differentially expressed. These distinct astrocyte zones will mean

\section{Infarct Core Peri-Infarct Brain Tissue}

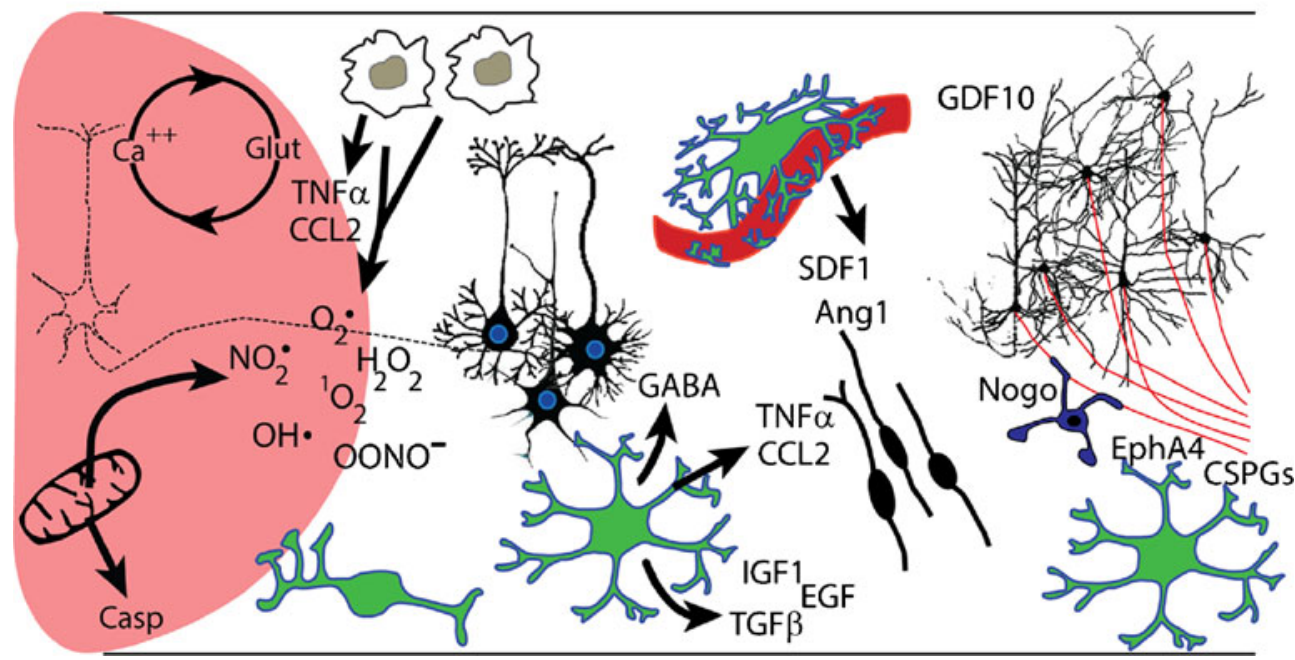

Fig. 2 The radial and regenerative progression of stroke can be collapsed in time to mock up the initial events of stroke damage and how these lead to later processes of partial tissue repair in adjacent peri-infarct cortex. Glut $=$ glutamate; $\mathrm{TNF} \alpha=$ tumor necrosis factor $-\alpha$; CLL $2=$ chemokine
(C-C motif) ligand 2; Casp = caspase; GABA $=\gamma$-aminobutyric acid; SDF1 $=$ stromal-derived factor $1 ;$ Ang $1=$ angiopoietin 2 ; IGF1 = insulin-like growth factor $1 ; \mathrm{EGF}=$ endothelial growth factor; $\operatorname{TGF} \beta=$ transforming growth factor- $\beta$; CSPG $=$ chondroitin sulfate proteoglycan 
distinct regions of tissue repair and recovery and are likely to be an important area of future study in the field.

\section{Relayed Stroke: Effects of Ischemia on Distant Connected Brain Regions}

The effects of focal stroke are relayed to distant brain sites through loss of connections, secondary axonal degeneration, and microglial and astrocyte activation. Stroke first transmits electrical impulses that relay a damage signal to distant, connected brain regions. This produces an upregulation of immediate early genes within hours of the initial stroke, in areas that are clearly not exposed to the ischemia [78]. Stroke in one site causes the axons projecting from the now-dead neurons to lose their synaptic connections to distant sites. The axons from neurons that were in the stroke core will die. Dying of axons from a loss of neuronal cell bodies is termed Wallerian degeneration. Wallerian degeneration can be seen clinically in MRI in long axonal tracts as an early diminished diffusion of water and later as hyperintensities in T2 or FLAIR sequences, corresponding to periods of initial axon damage and then degeneration [79-81]. Dying axons release signals that cause reactive astrocytosis and microglial responses within days to weeks of the distant stroke [78, 81]. These distant signals from a focal stroke are detected at connected sites to the stroke core, such as the contralateral cortex, substantia nigra, brain stem, and spinal cord, and include activation of the cytokines TNF- $\alpha$ and interleukin-6 [78]. In the spinal cord after focal cortical stroke, TNF- $\alpha$ and interleukin- 6 are induced within the first 2 weeks of stroke, as well as the growth factors brainderived neurotrophic factor and neurotrophin 3 [82]. These distant sites are clearly not in the initial area of ischemia, and reflect the relayed nature of stroke. The induction of growth factor signaling at distant sites from stroke, such as the spinal cord, may be part of the mechanism for axonal sprouting in these sites.

These distant effects of stroke might be construed as diaschisis. Diaschisis in its original, definitional form refers to electrical dysfunction remote from a focal lesion. It later came to mean metabolic dysfunction or a reduction in blood flow in remote brain areas after a single focal stroke [83, 84]. Recent network analyses in human and animal models of stroke have shown widespread disconnection of functional brain networks with stroke. Similar to the above neuroanatomical and molecular biological effects of stroke on distant, connected brain regions, stroke disconnects brain areas that are connected in sensorimotor, language, attentional, and memory-related systems $[85,86]$. However, this is not diaschisis. These disruptions in neuronal networks by stroke occur in regions in which metabolic or blood flow changes have not been reported in focal stroke. Recent attempts to reconcile the historical concept of diaschisis with newer findings that many, many brain areas are disconnected by stroke has led to the awkward expansion of this term into "focal diaschisis", "connectional diaschisis", and "connectomal diaschisis" [84].

At its heart and in the original coining of the term, diaschisis was used to explain why focal stroke could produce behavioral deficits not associated with the location of the stroke $[83,84]$. In recent studies of widespread neuronal network alterations after single strokes, behavioral deficits occur as a result of loss of connections between brain areas in many patterns, which are far more diverse in their connectivity than could be explained by known patterns of diaschisis [86]. For example, in aphasia disintegration in the connectivity of a distributed network of cortical areas well outside the traditional left frontal and parietal language areas can produce language deficits, or in memory function loss of connectivity among many frontal, thalamic, and medial temporal sites can produce deficits in stroke - in each function (language, memory) disruptions in many different anatomical networks can produce the same type of behavioral deficit. This is well beyond the original concept of diaschisis of a remote metabolic disturbance in one brain region produced from a stroke in another. Similarly, as noted above, neuroanatomical (astrocytosis) and molecular (cytokine induction) alterations occur after focal stroke in distant areas such as thalamus, spinal cord, and red nucleus in which diaschisis has not been observed in cortical or forebrain stroke. Instead, these represent disconnection effects that are more widespread and not accompanied by metabolic or blood flow abnormalities. This choice of "disconnection" over "diaschisis" fits with the emerging concepts of neuronal network disintegration in stroke and is free from historical context (or baggage). Focal stroke disconnects: it produces distant changes in connected networks and these disrupt the function of these networks, as well as activating cellular and molecular signaling events within them, relayed from the stroke site.

The relayed nature of stroke also causes cell death. A delayed apoptotic cell death occurs in the thalamus in experimental stroke models after cortical stroke that is distant to the thalamus [87], and similarly in the substantia nigra after striatal stroke that is distant from the substantia nigra [81]. After white matter stroke, connected cortical areas suffer a delayed damage that is seen as cortical thinning in magnetic resonance imaging [88], and as an overall brain atrophy [89]. In the aged brain with white matter stroke there is increased microglial activation and inflammatory cytokine release in connected cortical areas, compared with the young adult [90]. In summary, stroke relays its effects throughout the brain along its network of connections. This relay occurs through initial electrical and molecular signals early after injury and then through delayed signals from degenerating axons. These signals induce cytokines and growth factors in contralateral cortex, basal ganglia, thalamus, brain stem, and spinal cord; 
activate astrocytes and microglia; and in some areas induce delayed neuronal degeneration. These relayed signals from stroke also induce tissue regeneration, discussed in the next section.

\section{Regenerative Stroke: Tissue Repair After Focal Ischemia}

\section{Neural Circuits in Stroke Recovery}

Stroke induces a limited process of repair and recovery. This is evidenced by axonal sprouting and the formation of new connections, angiogenesis and vascular remodeling, and in glial and neuronal progenitor responses. Progenitor responses occur in local peri-infarct tissue [29, 91]. Axonal sprouting occurs in peri-infarct tissue and from contralateral cortex from the stroke [56, 92-94]. From the perspective of time after stroke, the signals that occur with radial stroke trigger the cellular events that underlie regenerative stroke [95].

Axonal sprouting occurs from neurons that are in the cortex ipsilateral to the stroke and from neurons that are contralateral to the stroke. The location of axonal sprouting after stroke and its contribution to functional recovery likely depend on the stroke size. Small-to-medium-sized strokes occur in a brain hemisphere in which there is substantial remaining tissue adjacent to the stroke site. In experimental studies in rodent and nonhuman primate with these types of strokes, neurons from robust new connections in peri-infarct cortex, such as from motor cortex to somatosensory, premotor, prefrontal, and association areas $[39,52,69,96]$. These mediate motor recovery [36, 39]. In large strokes, there is a reduced amount of cortical tissue preserved in the ipsilateral hemisphere, and the cortex contralateral to the stroke exhibits axonal sprouting [97]. This axonal sprouting occurs from local axons in the spinal cord, as they grow into the regions of spinal cord and brain stem that lost their projections from the stroke site [92, 97, 98]. These connections also mediate a degree of motor recovery after stroke [97].

The molecular programs that underlie poststroke axonal sprouting are unique tissue regeneration events [36, 69]. Studies of the gene expression profile of sprouting neurons in stroke indicate that stroke activates a molecular program that has a coordinated pattern of signaling from the extracellular environment into the cell $[36,69,98]$. This includes specific growth factors and cytokines, cell surface receptors, intermediate cytoplasmic cascades, and transcriptional and epigenetic control points. These molecular programs differ between aged and young adult brains, and change with time after stroke. There is an early upregulation of a molecular induction program for axonal sprouting within the first week after stroke, and then a later maintenance program in axonal sprouting at 3 weeks after stroke [69]. A trigger for poststroke axonal sprouting is present within the first week after stroke. This distinction between an induction and maintenance program can be seen in the fact that extracellular signaling molecules, transcription factor, and epigenetic control molecules are activated in the induction phase, whereas cytoskeletal and synaptic proteins are more associated with the later, maintenance phase of axonal sprouting [69].

Several clinically relevant questions can be applied to our understanding of this poststroke axonal sprouting transcriptome. Does it differ in the aged brain? This is a key question for human stroke, as most strokes occur in aged individuals. Does the molecular program of axonal sprouting after stroke resemble that seen in the initial axonal sprouting that underlies neuronal development? Is there a trigger or triggers induced by stroke that actually set off this whole process of axonal sprouting and recovery?

The molecular control of axonal spouting is different in aged versus young adults. In directly comparing the transcriptional profile of sprouting neurons after stroke during the induction period of this response, there is little overlap of the young adult and aged sprouting transcriptome. Specifically, after correcting these transcriptional data sets for statistical significance, 1346 genes are differentially up- or downregulated in young adult sprouting neurons (neurons that form a new connection in 2-month-old rats after stroke) during the inductive phase after stroke. In total, 671 genes are up- or downregulated in aged sprouting neurons (sprouting neurons in stroke in 2-year-old rats). When these 2 data sets are directly compared, only 79 genes are commonly regulated by stroke in both young adult and aged sprouting neurons [69]. This indicates that in terms of tissue regeneration, stroke in the aged brain represents a very distinct biological event.

An open question in the neural repair field is whether regeneration recapitulates development. When the transcriptional profile of sprouting neurons after stroke is directly compared with that of neurons during development, there is a substantial statistical difference between these 2 molecular programs. In fact, if unsupervised genome-wide association analysis is applied to 180 different transcriptomes from the literature, from neurodevelopment, to learning and memory paradigms to spinal cord and other injuries, the greatest distinction is between neurons that have been induced into an axonal sprouting state after stroke and early postnatal neurons that are still forming new connections [36]. These studies indicate that neural repair does not strictly follow neural development on a molecular level.

The process of axonal sprouting after stroke is a profound biological event for the adult brain. In a brain region that normally does not form such substantial new connections, a process is triggered in which local and long distance projections are formed. This implies that there is a molecular trigger for this event. Working with candidate signaling molecules that are present in the aged neuron poststroke sprouting transcriptome, such a 
trigger was recently identified. The TGF- $\beta$ family member GDF10, which previously did not have a known role in brain function, was found to trigger axonal sprouting in vitro in many types of neurons, and in vivo after stroke, and to enhance functional recovery. The data identify GDF10 as a novel, stroke-induced brain growth factor. Interestingly, when endogenous levels of GDF10 are reduced, axonal sprouting after stroke is prevented, and motor recovery is reduced [36]. GDF10 signals through TGF- $\beta$ RI and II and Smad $2 / 3$, to activate PI3 kinase gene systems and to inhibit the signaling of phosphatase and tensin homolog and suppressor of cytokine signaling 3 . These gene systems mediate axonal sprouting in other contexts in the adult, such as in optic nerve and spinal cord injury [99-101]. These data indicate that GDF10 is one molecule in a trigger after stroke and coordinately activates parallel growth promotion cascades.

It is important to bring forward a word of caution on poststroke axonal sprouting. As noted above, in distinct animal models poststroke axonal sprouting is causally associated with motor recovery. Axonal sprouting is also a generalized phenomenon during recovery after stroke, in rats, mice, nonhuman primate,s and, by marker, in peri-infarct cortex in human stroke [102]. However, axonal sprouting in the adult brain is also associated with epilepsy. This is clearly the case with hippocampal axonal sprouting [103]. Axonal sprouting in animal models occurs within confined neuronal networks, such as premotor/motor/somatosensory areas, suggesting a functional relevancy and limited nature of this process. However, when behavioral activity is manipulated by forcing a stroke-affected animal to overuse its affected forelimb, and axonal growth inhibitors are blocked, axonal sprouting can occur from peri-infarct tissue into widespread areas of the cortical hemisphere, including orbitofrontal and temporal cortex, that are well outside of confined functional domains [39]. Similarly, when Nogo signaling is blocked after stroke at the same time that rehabilitation therapies are given, axonal sprouting is exuberant and aberrant and recovery is reduced [97]. Thus, poststroke axonal sprouting may have positive or negative effects depending on behavioral context. The clinical implication of this double-edged sword is that medicines that induce axonal sprouting after stroke, once available, will need to be carefully utilized with neurorehabilitation and screened for side effects.

\section{Tissue Regeneration After Stroke}

Outside of axonal sprouting, there are several other cellular events after stroke that are even more directly regenerative in that they produce new populations of neurons and blood vessels within a unique tissue microenvironment. Ischemia induces proliferation and migration of neural and glial progenitors. For neural progenitors, the largest germinal matrix in the brain is the subventricular or subependymal zone (SVZ). This lies close to the lateral ventricles. Multipotent neural progenitors (which can give rise to glia and neurons) divide to produce more rapidly proliferating cells (termed transit amplifying cells), which then give rise to immature neurons (termed neuroblasts). In the rodent brain, neuroblasts from the SVZ migrate to the olfactory bulb $[52,103]$. In the human brain, this migration to the olfactory bulb may be reduced, and neuroblasts may migrate into the basal ganglia [104]. Stroke signals to the SVZ, principally to transit amplifying cells, which proliferate, and to neuroblasts, which migrate to areas of damage. This response can produce new neurons in areas of damage [29, 30, 91]. If the stroke occurs in close proximity to the SVZ, such as in the striatum/basal ganglia, neurogenesis is robust and large numbers of neuroblasts migrate to areas adjacent to the stroke site. Most of these die, but some do mature and form local and longdistance connections. If the stroke site is distant to the SVZ, such as in cortex, fewer neuroblasts migrate to areas of injury and these survive in vanishingly small numbers [29]. Poststroke neurogenesis is stimulated by cytokines in the peri-infarct tissue, particularly SDF-1 and angiopoietin 2 $[29,30]$. It has been difficult to determine definitively if poststroke neurogenesis has a causal role in functional recovery after stroke, and future work will need to determine if this process is truly a "neural repair" event.

The regenerative response after stroke also includes angiogenesis and the modification of the vascular tree. In early human studies, the degree of angiogenesis was correlated with the degree of recovery [105]. Angiogenesis and vascular remodeling occur most prominently in peri-infarct tissue [106], although there are changes in vascular structure in cortex contralateral to stroke [107]. Angiogenesis initiates early after stroke, with changes in gene expression that underlie angiogenesis within days of stroke [108]. It is important to note that animal models of stroke differ in their initiation of angiogenesis. The photothrombotic stroke model, which is used in many optogenetic studies of stroke neural repair, does not produce substantial angiogenesis [65, 109]. However, other stroke models with reperfusion and the production of a periinfarct region of partial damage do induce angiogenesis [29, 107, 110]. Angiogenesis serves to bring new blood flow to peri-infarct tissue, but this occurs too late for an actual survival effect in the evolution of stroke damage. Instead, angiogenic vessels secrete growth factors, proteases, and cytokines that modulate the local tissue environment $[18,22]$. Angiogenesis is causally linked to neurogenesis in peri-infarct tissue and these vessels are the source for angiopoietin 2 and SDF-1, which induce migration of neuroblasts after stroke [29]. In spinal cord injury, angiogenic vessels are the source of eicosanoids that induce axonal sprouting [22]. 
However, there has been no direct link between axonal sprouting and angiogenesis in stroke.

\section{Potential Neural Repair Therapies}

The biological findings in regenerative stroke suggest targets for neural repair therapies. In keeping with the neural circuit theme of this issue, stem cell, biologic, and small molecule therapies have been tested for their role in axonal sprouting in preclinical models of stroke. A comprehensive discussion of these therapies is beyond the scope of this review. However, some general biological themes or pathways emerge from studies of neural repair after stroke. The transcriptome of sprouting neurons after stroke identifies several molecular pathways that might promote recovery, and excludes others. Blockade of Nogo receptor 1 (NgR1) but not Nogo receptor 2 (NgR2) promotes axonal sprouting after stroke [69]. NgR1 antagonists have been tested in many stroke models and found to be effective in promoting recovery [97, 111-113]. NgR2 binds myelin-associated glycoprotein (MAG) [114]. An antimyelin-associated glycoprotein antibody has recently been tested in a phase I trial in stroke [115]. The data from preclinical studies of axonal sprouting would suggest that this will not be effective, and preclinical models show that it is only modestly successful in either preventing the initial dysfunction after stroke or producing limited behavioral recovery [116]. Other molecular pathways for axonal sprouting, either induced in peri-infarct neurons or contralateral neurons [98], indicate promising targets for neural repair such as ephrin-A5, GDF10, and the use of the small molecule inosine [36, 69, 98, 113]. Cell therapies appear to stimulate local axonal sprouting by tissue immunohistochemical staining [117-119], and in some cases by more detailed direct demonstration of axonal connections [120]. These also represent a candidate neural repair approach.

\section{Conclusion}

Ischemic stroke occurs as a continuum in time of biological responses in the brain from initial ischemic cell death to secondary damage to regeneration and partial repair. Each element in this continuum is triggered by processes in the preceding stage. Stroke also occurs as a progressive, spatially evolving biological response in the brain, from the initial ischemic core to radial brain regions at successive distances to the core and then to brain areas that are distant but connected to the core. Putting these two concepts of a stroke continuum in time and in spatial progression together develops the three Rs of stroke: 1) radial-damage progresses locally through the brain and away from the infarct in distinct zones; 2) relayed - damage signals are relayed to connected brain areas both locally and distantly; 3 ) regeneration - damage signals create a tissue regenerative response that produces limited brain repair.

Acknowledgments This work was supported by grants NS085019, NS081055, NS077521, and NS071481; the American Stroke Association/Bugher Foundation UCLA Stroke Collaborative Research Center; the Richard Merkin Foundation for Neural Repair at UCLA; and the Dr. Miriam and Sheldon G. Adelson Medical Research Foundation.

\section{Compliance with Ethical Standards}

Required Author Forms Disclosure forms provided by the authors are available with the online version of this article.

\section{References}

1. Lackland DT, Roccella EJ, Deutsch AF, et al. American Heart Association Stroke Council; Council on Cardiovascular and Stroke Nursing; Council on Quality of Care and Outcomes Research; Council on Functional Genomics and Translational Biology. Factors influencing the decline in stroke mortality: a statement from the American Heart Association/American Stroke Association. Stroke 2014;45:315-353.

2. Center for Disease Control and Prevention. Stroke facts. Availabe at: http://www.cdc.gov/stroke/facts.htm. Accessed Month Day, 2015.

3. Koton S, Schneider AL, Rosamond WD, et al. Stroke incidence and mortality trends in US communities, 1987 to 2011. JAMA 2014;312:259-268.

4. Sharp FR, Lu A, Tang Y, Millhorn DE. Multiple molecular penumbras after focal cerebral ischemia. J Cereb Blood Flow Metab 2000;20:1011-1032.

5. Moskowitz MA, Lo EH, Iadecola C. The science of stroke: mechanisms in search of treatments. Neuron 2010;67:181-198.

6. Lai TW, Zhang S, Wang YT. Excitotoxicity and stroke: identifying novel targets for neuroprotection. Prog Neurobiol 2014;115:157188.

7. O'Collins VE, Macleod MR, Donnan GA, et al. 1,026 experimental treatments in acute stroke. Ann Neurol 2006;59:467-477.

8. Redzic ZB, Rabie T, Sutherland BA, Buchan AM. Differential effects of paracrine factors on the survival of cells of the neurovascular unit during oxygen glucose deprivation. Int $\mathrm{J}$ Stroke 2013;10:407-414.

9. Sozmen EG, Hinman JD, Carmichael ST. Models that matter: white matter stroke models. Neurotherapeutics 2012;9:349-358.

10. Mabuchi T, Kitagawa K, Ohtsuki T, et al. Contribution of microglia/macrophages to expansion of infarction and response of oligodendrocytes after focal cerebral ischemia in rats. Stroke 2000;31:1735-1743.

11. Chu XF, Fu XJ, Zou LY, et al. Oncosis, the possible cell death pathway in astrocytes after focal cerebral ischemia. Brain Res 2007; 1149:157-164.

12. Franke H, Illes P. Nucleotide signaling in astrogliosis. Neurosci Lett 2014;565:14-22.

13. Astrup J, Siesjo BK, Symon L. Thresholds in cerebral ischemiathe ischemic penumbra. Stroke 1981;12:723-725. 
14. Fisher M, Albers GW. Advanced imaging to extend the therapeutic time window of acute ischemic stroke. Ann Neurol 2013;73:49.

15. Davis S, Donnan GA. Time is penumbra: imaging, selection and outcome. The Johann Jacob Wepfer Award 2014. Cerebrovasc Dis 2014;38:59-72.

16. Schofield ZV, Woodruff TM, Halai R, Wu MC, Cooper MA. Neutrophils - a key component of ischemia-reperfusion injury. Shock 2013;40:463-470.

17. Morrison HW, Filosa JA. A quantitative spatiotemporal analysis of microglia morphology during ischemic stroke and reperfusion. J Neuroinflammation 2013;11;10-14

18. Zhoa B-Q, Tejima E, Lo EH. Neurovascular proteases in brain injury, hemorrhage and remodeling after stroke. Stroke 2007;38;748-752.

19. Gleichman AJ, Carmichael ST. Astrocytic therapies for neuronal repair in stroke. Neurosci Lett 2014;565:47-52.

20. Le Belle JE, Orozco NM, Paucar AA, et al. Proliferative neural stem cells have high endogenous ROS levels that regulate selfrenewal and neurogenesis in a PI3K/Akt-dependant manner. Cell Stem Cell 2011;7;8:59-71.

21. Hu Q, Liang X, Chen D, et al. Delayed hyperbaric oxygen therapy promotes neurogenesis through reactive oxygen species/hypoxiainducible factor- $1 \alpha / \beta$-catenin pathway in middle cerebral artery occlusion rats. Stroke 2014;45:1807-1814.

22. Brumm AJ, Carmichael ST. Not just a rush of blood to the head. Nat Med 2012;18:1609-1610.

23. Gertz K, Kronenberg G, Kälin RE, et al. Essential role of interleukin-6 in post-stroke angiogenesis. Brain 2012;135:19641980.

24. Carmichael ST, Chesselet MF. Synchronous neuronal activity is a signal for axonal sprouting after cortical lesions in the adult. J Neurosci 2002;22:6062-6070.

25. Iadecola C, Anrather J. The immunology of stroke: from mechanisms to translation. Nat Med 2011;17:796-808.

26. Benakis C, Garcia-Bonilla L, Iadecola C, Anrather J. The role of microglia and myeloid immune cells in acute cerebral ischemia. Front Cell Neurosci 2015;8:461.

27. Ekdahl CT, Kokaia Z, Lindvall O. Brain inflammation and adult neurogenesis: the dual role of microglia. Neuroscience 2009;158: 1021-1029.

28. Zhao Y, Rempe DA. Targeting astrocytes for stroke therapy. Neurotherapeutics 2010;7:439-451.

29. Ohab JJ, Carmichael ST. Poststroke neurogenesis: emerging principles of migration and localization of immature neurons. Neuroscientist 2008;14:369-380.

30. Kahle MP, Bix GJ. Neuronal restoration following ischemic stroke: influences, barriers, and therapeutic potential. Neurorehabil Neural Repair 2013;27:469-478.

31. Liu XS, Zhang ZG, Zhang RL, et al. Chemokine ligand 2 (CCL2) induces migration and differentiation of subventricular zone cells after stroke. J Neurosci Res 2007;85:2120-2125.

32. Gonzalez-Perez O, Gutierrez-Fernandez F, Lopez-Virgen V, et al. Immunological regulation of neurogenic niches in the adult brain. Neuroscience 2012;226:270-281.

33. Yan YP, Sailor KA, Lang BT, Park SW, Vemuganti R, Dempsey RJ. Monocyte chemoattractant protein-1 plays a critical role in neuroblast migration after focal cerebral ischemia. J Cereb Blood Flow Metab 2007;27:1213-1224.

34. Withers GS, Higgins D, Charette M, Banker G. Bone morphogenetic protein-7 enhances dendritic growth and receptivity to innervation in cultured hippocampal neurons. Eur J Neurosci 2000;12: 106-116.

35. Ren J, Kaplan PL, Charette MF, Speller H, Finklestein SP. Time window of intracisternal osteogenic protein-1 in enhancing functional recovery after stroke. Neuropharmacology 2000;39: 860-865.

36. Li S Nie EH, Yin Y, Benowitz L, et al. GDF10 is a signal for axonal sprouting and functional recovery after stroke. Nat Neurosci 2015 Oct 26 [Epub ahead of print].

37. Carmichael ST, Archibeque I, Luke L, Nolan T, Momiy J, Li S. Growth-associated gene expression after stroke: Evidence for a growth-promoting region in peri-infarct cortex. Expt Neurol 2005;193:291-311.

38. Shen LH, Li Y, Gao Q, Savant-Bhonsale S, Chopp M. Downregulation of neurocan expression in reactive astrocytes promotes axonal regeneration and facilitates the neurorestorative effects of bone marrow stromal cells in the ischemic rat brain. Glia 2008;56: 1747-1754.

39. Overman JJ, Clarkson AN, Wanner IB, et al. A role for ephrin-A5 in axonal sprouting, recovery, and activity-dependent plasticity after stroke. Proc Natl Acad Sci U S A 2012;14;109:E2230E2239.

40. Heiss WD. The ischemic penumbra: how does tissue injury evolve? Ann N Y Acad Sci 2012;1268:26-34.

41. Pacher P, Beckman JS, Liaudet L. Nitric oxide and peroxynitrite in health and disease. Physiol Rev 2007;87:315-424.

42. Fujii H, Sato-Akaba H, Kawanishi K, Hirata H. Mapping of redox status in a brain-disease mouse model by three-dimensional EPR imaging. Magn Reson Med 2011;65:295-303.

43. Sauer H, Wartenberg M. Reactive oxygen species as signaling molecules in cardiovascular differentiation of embryonic stem cells and tumor-induced angiogenesis. Antioxid Redox Signal 2005;71423-1434.

44. Vieira HL, Alves PM, Vercelli A. Modulation of neuronal stem cell differentiation by hypoxia and reactive oxygen species. Prog Neurobiol 2011;93:444-455.

45. Ohab JJ, Fleming S, Blesch, A, Carmichael ST. A neurovascular niche for neurogenesis after stroke. J Neurosci 2006;26:1300713016.

46. Pietrobon D, Moskowitz MA. Chaos and commotion in the wake of cortical spreading depression and spreading depolarizations. Nat Rev Neurosci 2014;15:379-393.

47. von Bornstädt D, Houben T, Seidel JL, et al. Supply-demand mismatch transients in susceptible peri-infarct hot zones explain the origins of spreading injury depolarizations. Neuron 2015;85: $1117-1131$.

48. Gulati T, Won SJ, Ramanathan DS, et al. Robust neuroprosthetic control from the stroke perilesional cortex. J Neurosci 2015;35: 8653-8661.

49. Katz LC, Shatz CJ. Synaptic activity and the construction of cortical circuits. Science 1996;274:1133-1138

50. Stellwagen D, Shatz CJ. An instructive role for retinal waves in the development of retinogeniculate connectivity. Neuron 2002;33: 357-367.

51. Egorov AV, Draguhn A. Development of coherent neuronal activity patterns in mammalian cortical networks: common principles and local hetereogeneity. Mech Dev 2013;130:412-423.

52. Brown CE, Aminoltejari K, Erb H, Winship IR, Murphy TH. In vivo voltage-sensitive dye imaging in adult mice reveals that somatosensory maps lost to stroke are replaced over weeks by new structural and functional circuits with prolonged modes of activation within both the peri-infarct zone and distant sites. J Neurosci 2009;29:1719-1734

53. Lim DA, Alvarez-Buylla A. Adult neural stem cells stake their ground. Trends Neurosci 2014;37:563-571.

54. Buma FE, Lindeman E, Ramsey NF, Kwakkel G. Functional neuroimaging studies of early upper limb recovery after stroke: a systematic review of the literature. Neurorehabil Neural Repair 2010;24:589-608. 
55. Heiss WD. WSO Leadership in Stroke Medicine Award Lecture Vienna, September 26, 2008: functional imaging correlates to disturbance and recovery of language function. Int J Stroke 2009;4: 129-136.

56. Murphy TH, Corbett D. Plasticity during stroke recovery: from synapse to behaviour. Nat Rev Neurosci 2009;10:861-872.

57. Lim DH, LeDue JM, Mohajerani MH, Murphy TH. Optogenetic mapping after stroke reveals network-wide scaling of functional connections and heterogeneous recovery of the peri-infarct. J Neurosci 2014:34:16455-16466.

58. Clarkson AN, Huang BS, Macisaac SE, Mody I, Carmichael ST. Reducing excessive GABA-mediated tonic inhibition promotes functional recovery after stroke. Nature 2010;468:305-309

59. Glykys J, Mody I. Activation of GABAA receptors: views from outside the synaptic cleft. Neuron 2007;56:763-770

60. Grutzendler J, Kasthuri N, Gan WB. Long-term dendritic spine stability in the adult cortex. Nature 2002;420:812-816.

61. Trachtenberg JT, Chen BE, Knott GW, et al. Long-term in vivo imaging of experience-dependent synaptic plasticity in adult cortex. Nature 2002;420:788-794.

62. Cheetham CE, Hammond MS, McFarlane R, Finnerty GT. Altered sensory experience induces targeted rewiring of local excitatory connections in mature neocortex. J Neurosci 2008;28: 9249-9260.

63. Jasinska M, Siucinska E, Cybulska-Klosowicz A, et al. Rapid, learning-induced inhibitory synaptogenesis in murine barrel field. J Neurosci 2010;30:1176-1184.

64. Fu M, Zuo Y. Experience-dependent structural plasticity in the cortex. Trends Neurosci 2011;34:177-187.

65. Mostany R, Chowdhury TG, Johnston DG, Portonovo SA, Carmichael ST, Portera-Cailliau C. Local hemodynamics dictate long-term dendritic plasticity in peri-infarct cortex. J Neurosci 2010;30:14116-14126.

66. Hinman JD, Rasband MN, Carmichael ST. Remodeling of the axon initial segment after focal cortical and white matter stroke. Stroke 2013;44:182-189.

67. Brown CE, Boyd JD, Murphy TH. Longitudinal in vivo imaging reveals balanced and branch-specific remodeling of mature cortical pyramidal dendritic arbors after stroke. J Cereb Blood Flow Metab 2010;30:783-791.

68. Carmichael ST, Wei L, Rovainen CM, Woolsey TA New patterns of intra-cortical connections after focal stroke. Neurobiol Dis 2001;8:910-922.

69. Li S, Overman JJ, Katsman D, et al. An age-related sprouting transcriptome provides molecular control of axonal sprouting after stroke. Nat Neurosci 2010;13:1496-1504.

70. Wanner IB, Anderson MA, Song B, et al. Glial scar borders are formed by newly proliferated, elongated astrocytes that interact to corral inflammatory and fibrotic cells via STAT3-dependent mechanisms after spinal cord injury. J Neurosci 2013;33:12870-12886.

71. Khahk BS, Sofroniew M. Diversity of astrocyte functions and phenotypes in neural circuits. Nat Neurosci 2015;18:942-952.

72. Barreto GE, Sun X, Xu L, Giffard RG. Astrocyte proliferation following stroke in the mouse depends on distance from the infarct. PLoS One 2011;6:e27881.

73. Shimada IS, Borders A, Aronshtam A, Spees JL. Proliferating reactive astrocytes are regulated by Notch-1 in the peri-infarct area after stroke. Stroke 2011;42:3231-3237.

74. Sofroniew MV. Transgenic techniques for cell ablation or molecular deletion to investigate functions of astrocytes and other GFAP-expressing cell types. Methods Mol Biol 2012;814:531544.

75. Zamanian JL, Xu L, Foo LC, et al. Genomic analysis of reactive astrogliosis. J Neurosci 2012;32:6391-6410.

76. López-Valdés HE, Clarkson AN, Ao Y, et al. Memantine enhances recovery from stroke. Stroke 2014;45:2093-2100.
77. Garcia AD, Petrova R, Eng L, Joyner AL. Sonic hedgehog regulates discrete populations of astrocytes in the adult mouse forebrain. J Neurosci 2010;30:13597-13608.

78. Block F, Dihné M, Loos M. Inflammation in areas of remote changes following focal brain lesion. Prog Neurobiol 2005;75: 342-365.

79. Matsusue E, Sugihara S, Fujii S, Kinoshita T, Ohama E, Ogawa T. Wallerian degeneration of the corticospinal tracts: postmortem MR-pathologic correlations. Acta Radiol 2007;48:690-694.

80. DeVetten G, Coutts SB, Hill MD, et al. Acute corticospinal tract Wallerian degeneration is associated with stroke outcome. Stroke 2010;41:751-756.

81. Zhang J, Zhang Y, Xing S, Liang Z, Zeng J. Secondary neurodegeneration in remote regions after focal cerebral infarction: a new target for stroke management? Stroke 2012;43:1700-1705.

82. Sist B, Fouad K, Winship IR. Plasticity beyond peri-infarct cortex: spinal up regulation of structural plasticity, neurotrophins, and inflammatory cytokines during recovery from cortical stroke. Exp Neurol 2014;252:47-56.

83. Meyer JS, Obara K, Muramatsu K. Diaschisis. Neurol Res 1993;15: 362-266.

84. Carrera E, Tononi G. Diaschisis: past, present, future. Brain 2014;137:2408-2422.

85. He BJ, Snyder AZ, Vincent JL, Epstein A, Shulman GL, Corbetta $\mathrm{M}$, Breakdown of functional connectivity in frontoparietal networks underlies behavioral deficits in spatial neglect. Neuron 2007;53:905-918.

86. Corbetta M, Ramsey L, Callejas A, et al. Common behavioral clusters and subcortical anatomy in stroke. Neuron 2015;85:927941.

87. Wei L, Ying DJ, Cui L, Langsdorf J, Yu SP. Necrosis, apoptosis and hybrid death in the cortex and thalamus after barrel cortex ischemia in rats. Brain Res 2004;1022:54-61.

88. Duering M, Righart R, Wollenweber FA, Zietemann V, Gesierich B, Dichgans M. Acute infarcts cause focal thinning in remote cortex via degeneration of connecting fiber tracts. Neurology 2015;84:1685-1692.

89. Kloppenborg RP, Nederkoorn PJ, Grool AM, et al. SMART Study Group. Cerebral small-vessel disease and progression of brain atrophy: the SMART-MR study. Neurology 2012;79:2029-2036

90. Rosenzweig S, Carmichael ST. Age-dependent exacerbation of white matter stroke outcomes: a role for oxidative damage and inflammatory mediators. Stroke 2013;44:2579-2586.

91. Kernie SG, Parent JM. Forebrain neurogenesis after focal Ischemic and traumatic brain injury. Neurobiol Dis 2010;37: 267-274.

92. Benowitz LI, Carmichael ST. Promoting axonal rewiring to improve outcome after stroke. Neurobiol Dis 2010;37:259-266.

93. Starkey ML, Schwab ME. How plastic is the brain after a stroke? Neuroscientist 2014;20:359-371.

94. Overman JJ, Carmichael ST. Plasticity in the injured brain: more than molecules matter. Neuroscientist 2014;20:15-28.

95. Dancause N, Barbay S, Frost SB, et al. Extensive cortical rewiring after brain injury. J Neurosci 2005;25:10167-10179.

96. Clarkson AN, López-Valdés HE, Overman JJ, Charles AC, Brennan KC, Thomas Carmichael S. Multimodal examination of structural and functional remapping in the mouse photothrombotic stroke model. J Cereb Blood Flow Metab 2013;33:716-723.

97. Wahl AS, Omlor W, Rubio JC, et al. Neuronal repair. Asynchronous therapy restores motor control by rewiring of the rat corticospinal tract after stroke. Science 2014;344:1250-1255.

98. Zai L, Ferrari C, Subbaiah S, et al. Inosine alters gene expression and axonal projections in neurons contralateral to a cortical infarct and improves skilled use of the impaired limb. J Neurosci 2009;29:8187-8197. 
99. Sun F, Park KK, Belin S, et al. Sustained axon regeneration induced by co-deletion of PTEN and SOCS3. Nature 2011;480:372375 .

100. Liu J, Wang Y, Akamatsu Y, et al. Vascular remodeling after ischemic stroke: mechanisms and therapeutic potentials. Prog Neurobiol 2014;115:138-156.

101. Danilov CA, Steward O. Conditional genetic deletion of PTEN after a spinal cord injury enhances regenerative growth of CST axons and motor function recovery in mice. Exp Neurol 2015;266: 147-160.

102. Dudek FE, Sutula TP. Epileptogenesis in the dentate gyrus: a critical perspective. Prog Brain Res 2007;163:755-773.

103. Goldman SA, Chen Z. Perivascular instruction of cell genesis and fate in the adult brain. Nat Neurosci 2011;26;14:1382-1389

104. Ernst A, Alkass K, Bernard S, et al. Neurogenesis in the striatum of the adult human brain. Cell 2014;156:1072-1083.

105. Krupinski J, Kaluza J, Kumar P, Kumar S, Wang JM. Role of angiogenesis in patients with cerebral ischemic stroke. Stroke 1994;25:1794-1798.

106. Ng SC, de la Monte SM, Conboy GL, Karns LR, Fishman MC. Cloning of human GAP-43: growth association and ischemic resurgence. Neuron 1988;1:133-139.

107. Ergul A, Alhusban A, Fagan SC. Angiogenesis: a harmonized target for recovery after stroke. Stroke 2012;43:2270-2274.

108. Hayashi T, Noshita N, Sugawara T, Chan PH. Temporal profile of angiogenesis and expression of related genes in the brain after ischemia. J Cereb Blood Flow Metab 2003;23:166-180.

109. Tennant KA, Brown CE. Diabetes augments in vivo microvascular blood flow dynamics after stroke. J Neurosci 2013;33:1919419204

110. Young CC, Al-Dalahmah O, Lewis NJ, et al. Blocked angiogenesis in Galectin-3 null mice does not alter cellular and behavioral recovery after middle cerebral artery occlusion stroke. Neurobiol Dis 2014;63:155-164.

111. Lee JK, Kim JE, Sivula M, Strittmatter SM. Nogo receptor antagonism promotes stroke recovery by enhancing axonal plasticity. J Neurosci 2004;24:6209-6217.
112. Fang PC, Barbay S, Plautz EJ, Hoover E, Strittmatter SM, Nudo RJ. Combination of NEP 1-40 treatment and motor training enhances behavioral recovery after a focal cortical infarct in rats. Stroke 2010;41:544-549.

113. Zai L, Ferrari C, Dice C, et al. Inosine augments the effects of a Nogo receptor blocker and of environmental enrichment to restore skilled forelimb use after stroke. J Neurosci 2011;31:5977-5988.

114. Robak LA, Venkatesh K, Lee H, et al. Molecular basis of the interactions of the Nogo-66 receptor and its homolog NgR2 with myelin- Robak associated glycoprotein: development of NgROMNI-Fc, a novel antagonist of CNS myelin inhibition. J Neurosci 2009;29:5768-5783.

115. Cramer SC, Abila B, Scott NE, Simeoni M, Enney LA; MAG111539 Study Investigators. Safety, pharmacokinetics, and pharmacodynamics of escalating repeat doses of GSK249320 in patients with stroke. Stroke 2013;44:1337-1342.

116. Barbay S, Plautz EJ, Zoubina E, Frost SB, Cramer SC, Nudo RJ. Effects of postinfarct myelin-associated glycoprotein antibody treatment on motor recovery and motor map plasticity in squirrel monkeys. Stroke 2015;46:1620-1625.

117. Eckert MA, Vu Q, Xie K, Yu J, Liao W, Cramer SC, Zhao W. Evidence for high translational potential of mesenchymal stromal cell therapy to improve recovery from ischemic stroke. J Cereb Blood Flow Metab 2013;33:1322-1334.

118. Lemmens R, Steinberg GK. Stem cell therapy for acute cerebral injury: what do we know and what will the future bring? Curr Opin Neurol 2013;26:617-625.

119. Tajiri N, Acosta S, Portillo-Gonzales GS, et al. Therapeutic outcomes of transplantation of amniotic fluid-derived stem cells in experimental ischemic stroke. Front Cell Neurosci $2014 ; 8: 227$.

120. Andres RH, Horie N, Slikker W, et al. Human neural stem cells enhance structural plasticity and axonal transport in the ischaemic brain. Brain. 2011;134:1777-1789. 